\title{
Boundary dynamics and multiple reflection expansion for Robin boundary conditions
}

\author{
M. Bordag, ${ }^{1, *}$ H. Falomir, ${ }^{2, \dagger}$ E. M. Santangelo, ${ }^{2, \sharp}$ and D. V. Vassilevich ${ }^{1, \$}$ \\ ${ }^{1}$ University of Leipzig, Institute for Theoretical Physics, Augustusplatz 10/11, 04109 Leipzig, Germany \\ ${ }^{2}$ Departamento de Física, Universidad Nacional de La Plata, C.C. 67, 1900 La Plata, Argentina
}

(Received 9 November 2001; published 28 February 2002)

\begin{abstract}
In the presence of a boundary interaction, Neumann boundary conditions should be modified to contain a function $S$ of the boundary fields: $\left(\nabla_{N}+S\right) \phi=0$. Information on quantum boundary dynamics is then encoded in the $S$-dependent part of the effective action. In the present paper we extend the multiple reflection expansion method to the Robin boundary conditions mentioned above, and calculate the heat kernel and the effective action (i) for constant $S$, (ii) to the order $S^{2}$ with an arbitrary number of tangential derivatives. Some applications to symmetry breaking effects, tachyon condensation and a brane world are briefly discussed.
\end{abstract}

DOI: 10.1103/PhysRevD.65.064032

PACS number(s): 04.62.+v

\section{INTRODUCTION}

Recent years have seen a considerable increase of the interest in the relations between bulk and boundary dynamics. One of the most exciting applications of the subject is the tachyon condensation in open string theory. Modern interest in this mechanism of symmetry breaking is connected with the papers by Sen and Zwiebach [1], though certain ideas were developed much earlier (see, e.g., [2]). Now, many different methods are being used in this field. An extensive literature survey can be found in [3]. The sigma model approach [4] is probably the closest one to the technique of the present paper. The tachyon field enters the boundary term of the open string action, and, therefore, modifies the open string boundary conditions.

In the present paper we deal with a scalar theory on a manifold $\mathcal{M}, \operatorname{dim} \mathcal{M}=m$. Let the classical action be of the form

$$
\mathcal{S}(\phi)=\int_{\mathcal{M}} d^{m} x\left[(\nabla \phi)^{2}+V(\phi)\right]+\int_{\partial \mathcal{M}} d^{m-1} x \widetilde{V}(\phi),
$$

with two so far arbitrary potentials $V$ and $\widetilde{V}$. If $\mathcal{M}$ is an open string world surface, and if $\phi$ is the string coordinate ${ }^{1} X^{\mu}$, then the boundary potential $\widetilde{V}$ may be identified with the boundary tachyon: $\widetilde{V}(\phi):=T(X)$.

Let us split $\phi$ into its background part $\bar{\phi}$ and the quantum fluctuations $\varphi: \phi=\bar{\phi}+\varphi$. To calculate the one-loop effective action we have to keep the part of $\mathcal{S}$ which is quadratic in $\varphi$ :

$$
\mathcal{S}_{2}=\int_{\mathcal{M}} d^{m} x \varphi D \varphi+\int_{\partial \mathcal{M}} d^{m-1} x \varphi\left(-\nabla_{N}-S\right) \varphi,
$$

\footnotetext{
*Email address: Michael.Bordag@itp.uni-leipzig.de

†Email address: falomir@obelix.fisica.unlp.edu.ar

*Email address: mariel@obelix.fisica.unlp.edu.ar

$\S$ On leave from V.A. Fock Institute of Physics, St. Petersburg University, 198904 St. Petersburg, Russia. Email address: Dmitri.Vassilevich@itp.uni-leipzig.de

${ }^{1}$ The coordinates $X^{\mu}$ are scalars from the world surface point of view.
}

where $\nabla_{N}$ is covariant derivative with respect to the inward pointing unit normal. $D$ is a second order partial differential operator which depends on $\bar{\phi}, S=-\frac{1}{2} \widetilde{V}^{\prime \prime}(\bar{\phi})$; the prime denotes differentiation with respect to $\phi$. Boundary conditions for the fluctuations $\varphi$ follow from the requirement that the boundary part of the action (2) vanishes. These can be either Dirichlet $\left.\varphi\right|_{\partial \mathcal{M}}=0$ or Robin

$$
\left.\left(\nabla_{N}+S\right) \varphi\right|_{\partial \mathcal{M}}=0
$$

boundary conditions. Both sets of boundary conditions ensure also the Hermiticity of the operator $D$. In the present paper we will consider Robin boundary conditions only. We will be interested in the dependence on the function $S$ of the heat kernel coefficients, the trace of the heat kernel, and the one-loop effective action.

The brane-world scenario [5] usually assumes that there is an interaction of the bulk fields which is confined on a surface $\Sigma$. Many essential features of such interactions can be described by the action (1) where in the second term one integrates over the surface $\Sigma$. One-loop quantum corrections are then given by the determinant of the operator $D$ subject to the following matching conditions on $\Sigma$,

$$
\begin{gathered}
\varphi_{+}=\varphi_{-}, \\
-\left(\nabla_{N} \varphi\right)_{+}+\left(\nabla_{N} \varphi\right)_{-}+\frac{1}{2} \widetilde{V}(\bar{\phi}) \varphi=0,
\end{gathered}
$$

where the subscripts "+" and "-" denote limiting values on $\Sigma$ from the two sides of the surface. It has been demonstrated in $[6,7]$ that if the background fields are symmetric under the reflection about $\Sigma$, the $\widetilde{V}$-dependent part of the heat kernel (and, consequently, that of the effective action) is indeed described by the Robin boundary value problem (3) with $S=-\frac{1}{4} \widetilde{V}(\bar{\phi})$. Hence, the results for the effective action which we will obtain in this paper are valid also for the brane-world scalar field (though they will be, of course, modified by the presence of the background curvature).

These two examples - strings and brane-world - are the main physical motivations for our study. For this reason, our explicit calculations will be carried out with an emphasis on dimensions two and five. However, since the main purpose 
of this paper is to develop a technique, we will not go too far in the applications (and will not give a more detailed literature survey). We reserve these subjects for future publications. Robin boundary conditions have also been considered recently in the context of AdS conformal field theory (CFT) [8].

The heat kernel technique is by now a standard tool of quantum field theory. It allows to extract divergences and anomalies in a very efficient way, and to represent the one loop effective action in a nice geometric form. More details on related mathematics and physical applications may be found in the books [9]. Moreover, a knowledge of the effective potential allows one to study symmetry breaking effects with the formation of a (boundary) condensate. The imaginary part of the quantum potential tells us about vacuum instability, while the derivative part of the effective action is related to the momentum dependence of certain diagrams.

In previous papers $[6,10]$, we showed that the multiple reflection expansion [11] is a powerful tool for studying the asymptotics of the heat kernel, and applied it to some classical boundary problems, as well as singular potentials for second order operators on compact manifolds with spherical symmetry.

In the present paper, we apply the same technique to second order differential operators on flat $m$-dimensional manifolds, acting on functions that satisfy, at the $(m-1)$-dimensional boundary, the conditions in Eq. (3).

In Sec. II A, the general form of the smeared boundary heat kernel coefficients is obtained. In the particular case of a constant field $S$, and after taking the smearing function to unity, the resulting series is shown to be summable, and the complete boundary contribution to the trace of the heat kernel is then obtained for any dimension $m$ in Sec. II B.

Section II C contains the multiple reflection expansion study of the boundary contribution to the trace of the heat kernel in the case of a field $S$ depending on the boundary coordinates. In this situation, each order in the multiple reflection expansion is shown to give, not only the asymptotic contributions, but also the non-asymptotic ones.

Using these results, the boundary one-loop effective Lagrangian is evaluated for constant $S$ in Sec. III A. The boundary one-loop effective kinetic energy for a general $S(z)$ is studied in Sec. III B.
In Appendix A we show that our results for the trace of the heat kernel can be extended to curved boundaries. Appendix B contains some details on the treatment of possible negative eigenvalues. Explicit expressions for the boundary part of the Coleman-Weinberg potential for massive fields are given in Appendix C.

\section{HEAT KERNEL}

\section{A. Multiple reflection expansion}

In this paper we are interested in the dependence of the heat kernel and the effective action on boundary values of the background field $\bar{\phi}$, through the function $S$ in the boundary conditions (3). We perform actual calculations for a simple flat geometry. Our results must then be understood as an approximation to more generic situations.

Let $\mathcal{M}$ be a flat, $m$-dimensional half space, and $V(\phi)$ $=0$ in Eq. (1) (then $D$ is free scalar Laplacian). The heat kernel $K(x, y ; t)$ is defined as a solution of the heat equation

$$
\left(\partial_{t}+D_{x}\right) K(x, y ; t)=0
$$

with the initial condition $K(x, y ; 0)=\delta(x-y)$ inside the manifold. It must be supplemented with (Robin) boundary conditions when its first argument belongs to the boundary.

The heat kernel for Robin boundary conditions satisfies the Dyson equation

$$
\begin{aligned}
K(x, y ; t)= & K_{N}(x, y ; t) \\
& +\int_{0}^{t} d s \int_{\partial M} d z K_{N}(x, z ; s) S(z) K(z, y ; t-s),
\end{aligned}
$$

where $K_{N}$ is the heat kernel for Neumann boundary conditions $(S=0)$. It admits a solution in terms of a power series in $S$

$$
\begin{aligned}
K(x, y ; t)= & K_{N}(x, y ; t)+\sum_{n=1}^{\infty} \int_{0}^{t} d s_{n} \int_{0}^{s_{n}} d s_{n-1} \ldots \int_{0}^{s_{2}} d s_{1} \int_{\partial M} d z_{n} \ldots \\
& \times \int_{\partial M} d z_{1} \times K_{N}\left(x, z_{n} ; t-s_{n}\right) S\left(z_{n}\right) K_{N}\left(z_{n}, z_{n-1} ; s_{n}-s_{n-1}\right) \ldots S\left(z_{1}\right) K_{N}\left(z_{1}, y ; s_{1}\right),
\end{aligned}
$$

which is nothing but a multiple reflection representation for the heat kernel. It is, of course, equivalent to the multiple reflection representation for the propagator [11].

A very simple (and also very naive) way to derive Eqs. (6) and (7) is to consider the boundary interaction term $\int_{\partial M} \varphi S \varphi$ as a perturbation to the Neumann problem, represent the heat kernel as $K(x, y ; t)=\left[x\left|\exp \left(-t\left(D+S \delta_{\partial M}\right)\right)\right| y\right]$ and expand the exponential in a power series of $S$. This derivation ig- 
nores completely all existence and convergence requirements, but reproduces correctly the combinatorics of the expansion. Similar arguments fail for other types of perturbations. Equation (7) can be also derived from the corresponding equation in [6] using equivalence to semitransparent boundaries. Note that, after suitable modifications in the multiple reflection expansion, $S$ may be replaced by a general differential operator [12].

The representation (7) was used in the context of singular potentials in [6] to prove some general properties of the heat kernel and also in [13] to calculate the heat trace asymptotics.

Consider the smeared heat kernel

$$
K(f, t)=\int_{\mathcal{M}} d^{m} x f(x) K(x, x ; t) .
$$

This is a mathematically consistent way to deal with the distributional nature of the heat kernel diagonal. ${ }^{2}$ Consider- ing just the integrated heat kernel $(f=1)$ is insufficient for many physical applications where one needs local quantities (as, e.g., the trace anomaly). However, it can safely be taken equal to one when studying global quantities, such as the integrated heat kernel coefficients or the integrated $\zeta$ function. This will be the case for the applications to be considered in this paper.

Suppose $\mathcal{M}$ is a manifold of product type so that, near the boundary, it can be decomposed as $\partial M \times[0, \delta]$. Let $x$ $=(z, r)$, where $z$ is a coordinate on the boundary, and $r$ is a normal coordinate. The smearing function $f$ can be expanded in a Taylor series in $r$ :

$$
f(z, r)=\sum_{p=0}^{\infty} \frac{r^{p}}{p !} f^{(p)}(z),
$$

where $f^{(p)}(z)$ denotes the boundary value of the $p$-th normal derivative. By substituting Eqs. (7) and (9) in Eq. (8) and integrating over $r$ we obtain

$$
\begin{aligned}
K(f, t)= & \sum_{n=0}^{\infty} \sum_{p=0}^{\infty} \int_{\partial M} d z f^{(p)}(z) \frac{2^{p}}{p !} \Gamma\left(\frac{1+p}{2}\right) \int_{0}^{t} d s_{n} \int_{0}^{s_{n}} d s_{n-1} \ldots \int_{0}^{s_{2}} d s_{1} \\
& \times\left(\frac{\left(t-s_{n}\right) s_{1}}{t-s_{n}+s_{1}}\right)^{(1+p) / 2} \int_{\partial M} d z_{n} \ldots \int_{\partial M} d z_{1} K_{N}\left(z, z_{n} ; t-s_{n}\right) S\left(z_{n}\right) K_{N}\left(z_{n}, z_{n-1} ; s_{n}-s_{n-1}\right) \ldots S\left(z_{1}\right) K_{N}\left(z_{1}, z ; s_{1}\right),
\end{aligned}
$$

where we have used that

$$
\begin{gathered}
K_{N}\left(x, z_{i} ; s\right)=\exp \left(-r^{2} / 4 s\right) K_{N}\left(z, z_{i} ; s\right), \\
x=(z, r) .
\end{gathered}
$$

Strictly speaking, the represenation (10) is valid on the product manifold, $\mathcal{M}=\partial \mathcal{M} \times[0, \infty]$. However, if one is interested in the small $t$ asymptotics of the heat kernel (which is local), the form of the interior becomes irrelevant, and Eq. (10) can be used to calculate the $S$-dependent contributions on an almost arbitrary manifold. If there is a parameter which damps long-range correlations (as mass, for example), our results become approximately valid for the effective action as well. The quality of such an approximation must be checked in each individual case.

To study the Casimir interaction between two Robin boundaries [14] one must replace Eq. (11) by the Neumann heat kernel in the strip (which can be also presented in a closed form).

\footnotetext{
${ }^{2}$ Indeed, the small $t$ asymptotics of the integral (8) contains boundary integrals with normal derivatives of $f$ (see, e.g., Gilkey [9]), thus indicating the presence of $\delta$-function terms.
}

To evaluate Eq. (10) in the case of a general, $z$-dependent $S$, it will prove convenient to make a Fourier transformation

$$
\begin{aligned}
K_{N}\left(z_{i}, z_{i-1} ; s\right)= & \int \frac{d^{m-1} k_{i}}{(2 \pi)^{(m-1) / 2}} \frac{1}{\sqrt{\pi s}} \\
& \times \exp \left(-s k_{i}^{2}-i\left(z_{i}-z_{i-1}\right) k\right) \\
S\left(z_{j}\right)= & \int d^{m-1} \widetilde{k_{j}} \widetilde{S}\left(\widetilde{k}_{j}\right) \exp \left(i \widetilde{k}_{j} z_{j}\right) \\
\widetilde{S}\left(\widetilde{k}_{j}\right)= & \int \frac{d^{m-1} z_{j}}{(2 \pi)^{m-1}} S\left(z_{j}\right) \exp \left(-i \widetilde{k}_{j} z_{j}\right) .
\end{aligned}
$$

However, we will first treat the case of a constant field, where it is not necessary to go to the Fourier-conjugate space.

\section{B. Heat kernel for constant $S$}

In this case, an individual term in the double sum in Eq. (10) reads: 


$$
\begin{array}{rl}
\int_{\partial \mathcal{M}} & d z f^{(p)}(z) S^{n} \frac{2^{p}}{p !} \Gamma\left(\frac{1+p}{2}\right) \int_{\partial \mathcal{M}} \ldots \int_{\partial \mathcal{M}} d z_{1} \ldots d z_{n} \pi^{-(n+1) / 2} \\
\quad \times \int_{0}^{t} d s_{n} \int_{0}^{s_{n}} d s_{n-1} \ldots \int_{0}^{s_{2}} d s_{1}\left(\frac{\left(t-s_{n}\right) s_{1}}{t-s_{n}+s_{1}}\right)^{(1+p) / 2} \int \prod_{i=0}^{n} \frac{d^{m-1} k_{i}}{(2 \pi)^{m-1}}\left[\left(t-s_{n}\right)\left(s_{n}-s_{n-1}\right) \ldots s_{1}\right]^{-1 / 2} \\
\quad \times \exp \left(-\left(t-s_{n}\right) k_{n}^{2}-\ldots-s_{1} k_{0}^{2}\right) \exp \left(-i k_{n}\left(z-z_{n}\right)-i k_{n-1}\left[z_{n}-z_{n-1}-\ldots-i k_{0}\left(z_{1}-z\right)\right]\right) .
\end{array}
$$

The integration over $z_{i}$ can be easily performed; it gives as a result

$$
\left[(2 \pi)^{m-1}\right]^{n} \delta\left(k_{n}-k_{n-1}\right) \delta\left(k_{n-1}-k_{n-2}\right) \ldots \delta\left(k_{1}-k_{0}\right) .
$$

These $\delta$-functions can be used to integrate over $k_{i}, i \neq 0$. Integration over $k_{0}$ gives then $(\pi / t)^{(m-1) / 2}$. After the change of variables $\alpha_{1}=s_{1} / t, \alpha_{2}=\left(s_{2}-s_{1}\right) / t, \ldots, \alpha_{0}=1-s_{n} / t$, the integration over $\{\alpha\}$ reduces to $I(p / 2,-(1+p) / 2,-1 / 2, \ldots$, $-1 / 2)$, where $I$ is a particular value of the following integral:

$$
I\left(A_{0}, A_{1}, \ldots, A_{n}\right)=\int_{\Sigma \alpha_{i}=1} \prod d \alpha_{i}\left(\alpha_{0} \alpha_{1}\right)^{A_{0}}\left(\alpha_{0}+\alpha_{1}\right)^{A_{1}} \prod_{i=2}^{n} \alpha_{i}^{A_{i}}=\frac{\Gamma\left(2 A_{0}+A_{1}+2\right) \Gamma\left(A_{0}+1\right)^{2} \prod_{i=2}^{n} \Gamma\left(A_{i}+1\right)}{\Gamma\left(2 A_{0}+2\right) \Gamma\left(A_{0}+\sum_{i=0}^{n} A_{i}+n+1\right)} .
$$

The final result for (13) reads:

$$
\frac{1}{(4 \pi)^{(m-1) / 2}} \int_{\partial \mathcal{M}} d z f^{(p)}(z) S^{n} t^{1 / 2(n+p-m+1)} \frac{2^{-p-1}}{\Gamma\left(\frac{n+p}{2}+1\right)} .
$$

Note that each order in reflections gives one complete order in powers of $t$.

For $n+p \leqslant 4$, this result can be checked against the expressions of [15-17]. For $p=0$ we reproduce the heat kernel expansion for a delta-potential in one dimension [18].

The power series with individual terms given by (16) can be summed up to give a closed expression for the heat kernel. Taking the smearing function, $f=1$, we obtain for its trace

$$
K(t)=\frac{V}{2(4 \pi t)^{(m-1) / 2}}\left[e^{S^{2} t} \operatorname{erf}(S \sqrt{t})+e^{S^{2} t}\right]
$$

where $V$ is the (infinite) volume of the boundary, and $\operatorname{erf}(S \sqrt{t})$ is the error function

$$
\operatorname{erf}(S \sqrt{t})=\frac{2}{\sqrt{\pi}} \int_{0}^{S \sqrt{t}} d \xi e^{-\xi^{2}}
$$

Now, consider the term with $n=2, p=0$ and $f=1$ in Eq.

In particular, for $m=2$, Eq. (17) can be seen to coincide with the trace of the heat kernel given, for example, in Ref. [19]. ever, the effect of a nonzero mass $(M)$ can easily be seen [for instance, from Eq. (7)] to reduce to multiplication of each term in the multiple reflection expansion of the heat kernel by $e^{-M^{2} t}$, thus leading to an overall factor in Eq. (17).

\section{Expansion to the order of two reflections for a $z$-dependent $S$}

As before, we will take $f=1$. The term corresponding to no reflection $(n=0)$ is independent of $S$. It is then given by Eq. (16), with $p=0, f=1$ and $n=0$.

As for the one-reflection contribution, from Eq. (13) it can easily be seen to be

$$
\begin{aligned}
K_{1} & =\frac{2^{1-m}}{\pi^{m / 2}} t^{(2-m) / 2} \int_{\partial \mathcal{M}} d z S(z) \\
& =\frac{2^{1-m}}{\pi^{m / 2}} t^{(2-m) / 2} \widetilde{S}(\widetilde{k}=0) .
\end{aligned}
$$
(10):
The result (17) is valid for a massless scalar field. How- 


$$
\begin{aligned}
K_{2}= & \left.\int_{\partial \mathcal{M}} d z \int_{\partial \mathcal{M}} d z_{1} \int_{\partial \mathcal{M}} d z_{2} \frac{1}{\pi} \int_{0}^{t} d s_{2} \int_{0}^{s_{2}} d s_{1}\left(\frac{\left(t-s_{2}\right) s_{1}}{t-s_{2}+s_{1}}\right)^{1 / 2}\left[\left(t-s_{2}\right)\left(s_{2}-s_{1}\right) s_{1}\right]^{-1 / 2} \int \frac{d k_{1} d k_{2} d k_{3} d \widetilde{k}_{1} d \widetilde{k}_{2}}{(2 \pi)^{3(m-1)}} \widetilde{S}_{\left(k_{1}\right)}\right) \widetilde{S}\left(\widetilde{k}_{2}\right) \\
& \times \exp \left(-k_{1}^{2}\left(t-s_{2}\right)-k_{2}^{2}\left(s_{2}-s_{1}\right)-k_{3}^{2} s_{1}\right) \exp \left(-i k_{1}\left(z-z_{2}\right)-i k_{2}\left(z_{2}-z_{1}\right)-i k_{3}\left(z_{1}-z\right)+i \widetilde{k}_{1} z_{1}+i \widetilde{k}_{2} z_{2}\right) .
\end{aligned}
$$

Integration over $z_{1}$ and $z_{2}$ gives rise to $(2 \pi)^{2(m-1)} \delta\left(k_{2}-k_{3}+\widetilde{k}_{1}\right) \delta\left(k_{1}-k_{2}+\widetilde{k}_{2}\right)$. These $\delta$-functions are then used to integrate over $k_{1}$ and $k_{3}$. Next, we shift $k_{2}$ to complete the square in the exponential and integrate over (shifted) $k_{2}$. We thus obtain

$$
\begin{aligned}
K_{2}= & \int_{\partial \mathcal{M}} d z \frac{1}{\pi} \int_{0}^{t} d s_{2} \int_{0}^{s_{2}} d s_{1}\left[\left(t-s_{2}+s_{1}\right)\left(s_{2}-s_{1}\right)\right]^{-1 / 2} \frac{1}{(4 \pi t)^{(m-1) / 2}} \int d \widetilde{k}_{1} d \widetilde{k}_{2} \widetilde{S}\left(\widetilde{k}_{1}\right) \widetilde{S}\left(\widetilde{k}_{2}\right) \exp \left(i z\left(\widetilde{k}_{1}+\widetilde{k}_{2}\right)\right) \\
& \times \exp \left(-\frac{1}{t}\left[\widetilde{k}_{1}^{2}\left(t-s_{1}\right) s_{1}+\widetilde{k}_{2}^{2}\left(t-s_{2}\right) s_{2}+2 \widetilde{k}_{1} \widetilde{k}_{2}\left(t-s_{2}\right) s_{1}\right]\right) .
\end{aligned}
$$

The integration over $d z$ can now be performed, which gives

$$
K_{2}=\frac{1}{\pi} \int_{0}^{t} d s_{2} \int_{0}^{s_{2}} d s_{1}\left[\left(t-s_{2}+s_{1}\right)\left(s_{2}-s_{1}\right)\right]^{-1 / 2} \frac{1}{(4 \pi t)^{(m-1) / 2}} \int d^{m-1} k \widetilde{S}(k) \widetilde{S}(-k) \exp \left(-\frac{k^{2}}{t}\left[\left(s_{2}-s_{1}\right)\left(t-s_{2}+s_{1}\right)\right]\right)
$$

or, after exponentiating the first factor

$$
K_{2}=\frac{1}{\pi(4 \pi t)^{(m-1) / 2}} \int d^{m-1} k \tilde{S}(k) \widetilde{S}(-k) \int_{0}^{t} d s_{2} \int_{0}^{s_{2}} d s_{1} \int_{0}^{\infty} d z \frac{z^{-1 / 2}}{\Gamma\left(\frac{1}{2}\right)} \exp \left(-\left(z+\frac{k^{2}}{t}\right)\left(s_{2}-s_{1}\right)\left(t-s_{2}+s_{1}\right)\right) .
$$

Calling $s_{1}^{\prime}=s_{2}-s_{1}$, and integrating by parts in $s_{2}$, one gets

$$
K_{2}=\frac{t^{2}}{\pi(4 \pi t)^{(m-1) / 2}} \int d^{m-1} k \widetilde{S}(k) \widetilde{S}(-k) \int_{0}^{\infty} d z \frac{z^{-1 / 2}}{\Gamma\left(\frac{1}{2}\right)} \int_{0}^{1} d s \exp \left(-\left(z+\frac{k^{2}}{t}\right) t^{2} s(1-s)\right) .
$$

After performing the integral in $z$, this last expression gives

$$
K_{2}=\frac{t^{(3-m) / 2}}{\pi(4 \pi)^{(m-1) / 2}} \int d^{m-1} k \tilde{S}(k) \tilde{S}(-k) \int_{0}^{1} d s \frac{(1-s)^{1 / 2}}{s^{1 / 2}} \exp \left(-t k^{2} s(1-s)\right) .
$$

Now, using its transformation properties under $s \rightarrow 1-s$, the last integral in Eq. (21) can be rewritten as

$$
\begin{aligned}
\int_{0}^{1} d s \frac{(1-s)^{1 / 2}}{s^{1 / 2}} \exp \left(-t k^{2} s(1-s)\right) & =\frac{1}{2} \int_{0}^{1} d s \frac{1}{(1-s)^{1 / 2} s^{1 / 2}} \exp \left(-t k^{2} s(1-s)\right)=\int_{0}^{1 / 4} d s \frac{1}{(1-4 s)^{1 / 2} s^{1 / 2}} \exp \left(-t k^{2} s\right) \\
& =\frac{\pi}{2} \exp \left(-t \frac{k^{2}}{8}\right) I_{0}\left(t \frac{k^{2}}{8}\right),
\end{aligned}
$$

where $I_{0}(x)$ is the modified Bessel function of order 0 . So, after replacing this into Eq. (21), the contribution of two reflections to the trace of the heat kernel is seen to be

$$
\begin{aligned}
K_{2}= & \frac{t^{(3-m) / 2}}{2(4 \pi)^{(m-1) / 2}} \int d^{m-1} k \widetilde{S}(k) \widetilde{S}(-k) \\
& \times \exp \left(-t \frac{k^{2}}{8}\right) I_{0}\left(t \frac{k^{2}}{8}\right) .
\end{aligned}
$$

This completes the calculation of the trace of the heat kernel to the order of two reflections. We stress that Eq. (22) is exact to this order, as it contains also all non-asymptotic contributions. As in the constant- $S$ case, for a massive field, each order in the multiple reflection expansion turns out to be multiplied by $e^{-M^{2} t}$.

By going back to coordinate integrals, and expanding in $t$, we can write a less compact but more explicit form of the heat kernel asymptotics: 


$$
\begin{aligned}
K_{2} \sim \sum_{l=0}^{\infty} \int_{\partial \mathcal{M}} d z S(z) \partial^{2 l} S(z) \\
\quad \times \frac{t^{-[(m-1) / 2]+1+2 l}}{(4 \pi)^{(m-1) / 2}} \frac{\Gamma\left(l+\frac{1}{2}\right) \Gamma\left(l+\frac{3}{2}\right)}{\pi l ! \Gamma(2 l+2)} .
\end{aligned}
$$

Note that, in contrast to Eq. (22), this expansion is asymptotic, and it cannot be used for large values of $t$.

\section{EFFECTIVE BOUNDARY ACTIONS}

The results of the previous section can be used to calculate some parts of the one-loop effective action induced on the boundary by the bulk fluctuations. In what follows we will, in fact, obtain the full one-loop effective action for a constant $S$ field, and its kinetic piece in the case of an $S$ field depending on the boundary coordinate. This will be done in the framework of the $\zeta$-function regularization scheme $[20,21]$. by

In this regularization scheme, the effective action is given

$$
W^{\mathrm{reg}}=-\frac{1}{2} \zeta^{D}(0)^{\prime}
$$

Here, $D$ is the operator defined by Eqs. (2) and (3). The $\zeta$-function $\zeta^{D}(s)$ is the Mellin transform of the trace of the heat kernel of the operator $D$ :

$$
\begin{aligned}
\zeta^{D}(s) & =\mu^{2 s} \operatorname{Tr}\left(D^{-s}\right) \\
& =\frac{\mu^{2 s}}{\Gamma(s)} \int_{0}^{\infty} d t t^{s-1} \operatorname{Tr}(\exp (-t D)) .
\end{aligned}
$$

The parameter $\mu$ with the dimension of a mass has been introduced to make the $\zeta$-function dimensionless. The resulting $\log (\mu)$ describes the renormalization ambiguity.

In order to avoid infrared divergences, we will study the zeta function for a massive bulk field, $M$ being its mass. As we will see, the limit $M \rightarrow 0$ is direct in some cases, while an analytic continuation of the zeta function is required before taking such limit in other cases. As already pointed out, the effect of this nonzero mass reduces to multiplication of each term in the trace of the heat kernel by $e^{-M^{2} t}$.

\section{A. Coleman-Weinberg potential on the boundary}

The Coleman-Weinberg potential is, by definition, the effective action for constant background fields. In this case, the expression (16) applies for the trace of the heat kernel.

Due to translational invariance, an overall divergence proportional to the volume of the boundary will appear in the effective action. This is not, however, an obstacle to the approach, since it is the Lagrangian density that has a physical meaning for Coleman-Weinberg type effective actions.

Before going to the actual calculation, let us explicitly rewrite the trace of the heat kernel for a massive bulk field

$$
K(t)=\frac{V}{2(4 \pi t)^{(m-1) / 2}}\left[e^{\left(S^{2}-M^{2}\right) t} \operatorname{erf}(S \sqrt{t})+e^{\left(S^{2}-M^{2}\right) t}\right] .
$$

For $S^{2}<M^{2}, K(t)$ decays exponentially when $t \rightarrow \infty$. On the other hand, for $S^{2}>M^{2}$, due to the asymptotic large-t behavior of the error function, $K(t)$ diverges exponentially in this limit when $S>0$, and its Mellin transform cannot be naively performed. This is due to the presence of eigenmodes of the form $e^{-S r}$, which satisfy Eq. (3) and fall off as $r \rightarrow$ $+\infty$. These modes eventually correspond to negative eigenvalues of $-\partial^{2}+M^{2}$, and their contribution to the $\zeta$ function must be explicitly calculated and added to the Mellin transform of the trace of the heat kernel, once the divergent contribution is subtracted from the last. As a consequence, as we will see later, for $S^{2}>M^{2}$ with $S>0$ the effective Lagrangian receives an imaginary contribution, and presents a sign ambiguity. So, let us consider the three different situations in a separate way.

$$
\text { 1. } S^{2}<M^{2}
$$

The zeta function can be easily calculated by Mellintransforming the expression (26) for the trace of the heat kernel. Before integrating over the proper time $t$, it is convenient to write the error function as an integral:

$$
e^{\left(S^{2}-M^{2}\right) t} \operatorname{erf}(S \sqrt{t})=2 S \sqrt{\frac{t}{\pi}} \int_{0}^{1} d \xi e^{-\left[M^{2}+\left(\xi^{2}-1\right) S^{2}\right] t} .
$$

Thus,

$$
\begin{aligned}
K(t)= & \frac{V}{2(4 \pi t)^{(m-1) / 2}}\left(2 S \sqrt{\frac{t}{\pi}} \int_{0}^{1} d \xi e^{-\left[M^{2}+\left(\xi^{2}-1\right) S^{2}\right] t}\right. \\
& \left.+e^{\left(S^{2}-M^{2}\right) t}\right)
\end{aligned}
$$

In this case, a closed expression for the $\zeta$-function in terms of the hypergeometric function can be given. However, such an expression is not very useful in practical calculations. By using Eqs. (24) and (28) the effective Lagrangian for the case of a massive bulk field can be evaluated in a very fast and efficient way in this case $\left(S^{2}<M^{2}\right)$. In fact, since the integral in Eq. (28) converges uniformly, the derivative with respect to $s$ can be performed, and it can be evaluated at $s$ $=0$ before actually doing the integral. The results for $m$ $=1,2,3,4,5$ are given in Appendix C. This case is not relevant to the study of the problem with $M=0$.

$$
\text { 2. } S^{2}>M^{2}, S>0
$$

As anticipated in the beginning of this subsection, our first task will be, in this case, to identify the exponentially growing part of the trace of the heat kernel (26). This can be done by writing $\operatorname{erf}(S \sqrt{t})=1-\operatorname{erfc}(S \sqrt{t})$, where 


$$
\operatorname{erfc}(S \sqrt{t})=\frac{2}{\sqrt{\pi}} \int_{S \sqrt{t}}^{\infty} d \xi e^{-\xi^{2}}
$$

is the complementary error function. From its behavior for large $t$, it is clear that the piece to be subtracted from the trace of the heat kernel is given by

$$
K_{d i v}(t)=\frac{V e^{\left(S^{2}-M^{2}\right) t}}{(4 \pi t)^{(m-1) / 2}} .
$$

In Appendix B we show that, as we have already commented, this piece comes from those modes of the Laplacian eventually becoming negative. So, once this piece is subtracted, the trace of the heat kernel reads

$$
K_{s u b}(t)=\frac{-V}{2(4 \pi t)^{(m-1) / 2}}\left[e^{\left(S^{2}-M^{2}\right) t} \operatorname{erfc}(S \sqrt{t})\right] .
$$

The Mellin transform of this quantity will give a first contribution, $\zeta_{1}^{D}(s)$, to the relevant $\zeta$ function $\left[\zeta^{D}(s)\right]$. A second one will come from the explicit contribution of the subtracted modes. Also in Appendix B, this contribution is shown to be given by

$$
\begin{aligned}
\zeta_{2}^{D}(s)= & \frac{V \mu^{2 s}\left(S^{2}-M^{2}\right)^{-s+(m-1) / 2}}{(4 \pi)^{(m-1) / 2} \Gamma\left(\frac{m-1}{2}\right)} \\
& \times\left[(-1)^{-s} \frac{\Gamma\left(\frac{m-1}{2}\right) \Gamma(1-s)}{\Gamma\left(\frac{m+1-2 s}{2}\right)}\right. \\
& \left.+\frac{\Gamma\left(s+\frac{1-m}{2}\right) \Gamma(1-s)}{\Gamma\left(\frac{3-m}{2}\right)}\right] .
\end{aligned}
$$

As regards $\zeta_{1}^{D}(s)$, it can be obtained by Mellintransforming Eq. (30), with the complementary error function written in integral form, and following the same steps as in the $S^{2}<M^{2}$ case. It is given by

$$
\begin{aligned}
\zeta_{1}^{D}(s)= & \frac{-V S \mu^{2 s}}{2(4 \pi)^{(m-1) / 2}} \frac{\Gamma\left(s-\frac{m}{2}+1\right)}{\Gamma(s)}\left[\frac{\left(S^{2}-M^{2}\right)^{(m / 2)-(1 / 2)-s}}{\pi S} \Gamma\left(s-\frac{m}{2}+\frac{1}{2}\right) \Gamma\left(\frac{m}{2}-s\right)-\frac{2 M^{m-2 s}}{S^{2} \pi^{1 / 2}(m-2 s)}\right. \\
& \left.\times F\left(\frac{1}{2}, 1,1+\frac{m}{2}-s, \frac{M^{2}}{S^{2}}\right)\right] .
\end{aligned}
$$

From the total $\zeta$ function, which is the sum of Eqs. (31) and (32), the effective action for $S^{2}>M^{2}$ can be obtained for any dimension $m$, and $S>0$. In the odd dimensional cases, where the $\Gamma$ in the denominator supplies a power of $s$, an analytic result for the effective Lagrangian can be obtained. However, for even dimensions, the derivative with respect to $s$ of the hypergeometric function can only be performed numerically. At variance with the case in Sec. III A 1, the derivative cannot be evaluated at $s=0$ before performing the integral, since the last extends to a noncompact interval. For completeness, we give the analytic result for the effective Lagrangian in the case $m=1$ in Appendix C.

Let us now discuss the limit $M=0$. The limit of Eq. (31) is direct. As regards Eq. (32), it is valid for $\operatorname{Re}(s)>(m$ $-1) / 2$. The zero mass limit can be taken by restricting, furthermore, to $\operatorname{Re}(s)<m / 2$ (this is due to the fact that the trace of the heat kernel behaves at infinity as a power, rather than a decaying exponential). In this strip of the $s$-plane, one has

$$
\begin{aligned}
\left.\zeta^{D}(s)\right\rfloor_{M=0}= & \frac{V \mu^{2 s}}{(4 \pi)^{(m-1) / 2}}\left\{\frac{\left(S^{2}\right)^{-s+(m-1) / 2}}{\Gamma\left(\frac{m-1}{2}\right)}\left[(-1)^{-s} \frac{\Gamma\left(\frac{m-1}{2}\right) \Gamma(1-s)}{\Gamma\left(\frac{m+1-2 s}{2}\right)}+\frac{\Gamma\left(s+\frac{1-m}{2}\right) \Gamma(1-s)}{\Gamma\left(\frac{3-m}{2}\right)}\right]\right. \\
& \left.-S^{m-2 s-1} \frac{\Gamma\left(s-\frac{m}{2}+\frac{1}{2}\right) \Gamma\left(s-\frac{m}{2}+1\right) \Gamma\left(\frac{m}{2}-s\right)}{2 \pi \Gamma(s)}\right\} .
\end{aligned}
$$


From this $\zeta$ function, the effective Lagrangian for $M=0$ and $S>0$ can be easily obtained for any dimension. We list the results for $m=1$ and $m=2$.

For $m=1$ we have:

$$
\mathcal{L}^{\mathrm{eff}}=-\frac{1}{2} \log \left(\frac{S^{2}}{\mu^{2}}\right) \pm i \pi .
$$

For $m=2$

$$
\mathcal{L}^{\mathrm{eff}}=-\frac{S}{2 \pi}\left[\log \left(\frac{4 S^{2}}{\mu^{2}}\right)-2\right] \pm \frac{i S}{2},
$$

where $\gamma$ is Euler's constant, and $\psi(x)$ is the zero order polygamma function.
As anticipated, a sign ambiguity arises in the effective Lagrangian, due to the existence of negative eigenvalues.

$$
\text { 3. } S^{2}>M^{2}, S<0
$$

In this case, the trace of the heat kernel behaves as $e^{-M^{2} t}$ times a power when $t \rightarrow \infty$, and a region in the $s$ plane exists where it can be Mellin-transformed. It is convenient to write it as

$$
K(t)=\frac{V}{2(4 \pi t)^{(m-1) / 2}}\left[e^{\left(S^{2}-M^{2}\right) t} \operatorname{erfc}(|S| \sqrt{t})\right] .
$$

The resulting $\zeta$ function can be retrieved from Eq. (32) by changing the overall sign and turning $S$ into $|S|$. Thus, it is given by

$$
\begin{aligned}
\zeta^{D}(s)= & \frac{V|S| \mu^{2 s}}{2(4 \pi)^{(m-1) / 2}} \frac{\Gamma\left(s-\frac{m}{2}+1\right)}{\Gamma(s)}\left[\frac{\left(S^{2}-M^{2}\right)^{(m / 2)-(1 / 2)-s}}{\pi|S|} \Gamma\left(s-\frac{m}{2}+\frac{1}{2}\right) \Gamma\left(\frac{m}{2}-s\right)-\frac{2 M^{m-2 s}}{S^{2} \pi^{1 / 2}(m-2 s)}\right. \\
& \left.\times F\left(\frac{1}{2}, 1,1+\frac{m}{2}-s, \frac{M^{2}}{S^{2}}\right)\right] .
\end{aligned}
$$

The comments made after Eq. (32) also apply to this case. The effective Lagrangian in the massive case is given, for $m$ $=1$ in Appendix C.

In the limit $M=0$ one obtains, for $S<0$

$$
\left.\zeta^{D}(s)\right\rfloor_{M=0}=\frac{V \mu^{2 s}}{2(4 \pi)^{(m-1) / 2}}|S|^{m-2 s-1} \frac{\Gamma\left(s-\frac{m}{2}+\frac{1}{2}\right) \Gamma\left(s-\frac{m}{2}+1\right) \Gamma\left(\frac{m}{2}-s\right)}{\pi \Gamma(s)}
$$

and the effective actions for $m=1$ and $m=2$ are as follows.

For $m=1$ we have

$$
\mathcal{L}^{\mathrm{eff}}=-\frac{1}{2} \log \left(\frac{S^{2}}{\mu^{2}}\right)
$$

For $m=2$

$$
\mathcal{L}^{\text {eff }}=\frac{|S|}{2 \pi}\left[\log \left(\frac{4 S^{2}}{\mu^{2}}\right)-2\right]
$$

Let us discuss some qualitative features of the potentials obtained above. These potentials have a very nontrivial structure, especially for $M \neq 0$, providing a lot of possibilities for the symmetry breaking with the formation of a boundary condensate. The presence of an imaginary part for $S>M$ indicates an instability of the "Robin phase," which may eventually decay to some other (Dirichlet?) phase. Here we see many similarities with the tachyon condensation and
$D$-brane formation in open string theory. We are going to address these questions in a separate publication.

\section{B. Effective kinetic energy}

In this section, we will evaluate the one-loop correction to the kinetic energy of the boundary field $S(z)$, due to the quantum fluctuations of the bulk scalar field $\phi$. Since we are interested only in the kinetic part of the effective action (or, equivalently, the propagator), it will be enough to consider the multiple reflection expansion to the order of two reflections (higher orders will contain higher powers of the field $S)$.

The term involving no reflection at the boundary will be ignored. In fact, it is $S$-independent and can always be eliminated through a redefinition of the cosmological constant on the brane.

The one-reflection contribution to the zeta function is seen, from Eq. (18), to be given by 


$$
\begin{aligned}
\zeta_{1}= & \frac{\mu^{m-2} 2^{1-m}}{\pi^{m / 2} \Gamma(s)} \widetilde{S}(\widetilde{k}=0) \int_{0}^{\infty} d t t^{s+[(2-m) / 2]-1} e^{-\left(M^{2} / \mu^{2}\right) t} \\
= & \frac{\mu^{m-2} \Gamma\left(s-\frac{(m-2)}{2}\right) 2^{1-m}}{\pi^{m / 2} \Gamma(s)} \widetilde{S}(\tilde{k}=0) \\
& \times\left(\frac{M^{2}}{\mu^{2}}\right)^{\{[(m-2) / 2]-s\}}
\end{aligned}
$$

$$
\text { for } \operatorname{Re}(s)>\frac{m-2}{2} \text {, }
$$

which can be meromorphically extended to the region $\operatorname{Re}(s)<(m-2) / 2$. The limit $M \rightarrow 0$ can be seen to vanish in this region. So, no contribution from one reflection will appear in the effective action, for any dimension $m$.

Let us now go to the 2-reflection contribution. From Eq. (22) the zeta function is seen to be given by

$$
\begin{aligned}
& \frac{\mu^{m-3}}{2(4 \pi)^{(m-1) / 2} \Gamma(s)} \int d^{m-1} k \widetilde{S}(k) \widetilde{S}(-k) \int_{0}^{\infty} d t t^{s+[(3-m) / 2]-1} \exp \left(-t\left(\frac{k^{2}}{8 \mu^{2}}+\frac{M^{2}}{\mu^{2}}\right)\right) I_{0}\left(t \frac{k^{2}}{8 \mu^{2}}\right) \\
& =\frac{\mu^{m-3} \Gamma\left(s+\frac{3-m}{2}\right)}{2(4 \pi)^{(m-1) / 2} \Gamma(s)} \int d^{m-1} k \tilde{S}(k) \widetilde{S}(-k)\left(\frac{k^{2}}{8 \mu^{2}}\right)^{-s+[(m-3) / 2]}\left(1+\frac{8 M^{2}}{k^{2}}\right)^{[(m-3) / 2]-s} \\
& \quad \times F\left(\frac{s}{2}+\frac{5-m}{4}, \frac{s}{2}+\frac{3-m}{4} ; 1 ; \frac{k^{4}}{\left(k^{2}+8 M^{2}\right)^{2}}\right) \text { for } \operatorname{Re}(s)>\frac{m-3}{2},
\end{aligned}
$$

where $F$ is the hypergeometric function. Now, from the properties of these functions, it is easy to see that it is only for $m$ $=2$ that the analytic extension must be performed before the zero mass limit. For $m \geqslant 3, M$ can be taken to zero from the beginning.

Let us look at two interesting cases.

$$
\text { 1. } m=2
$$

In this case

$$
\begin{aligned}
\zeta_{2}= & \frac{\mu^{-1} \Gamma\left(s+\frac{1}{2}\right)}{2(4 \pi)^{1 / 2} \Gamma(s)} \int d^{m-1} k \tilde{S}(k) \widetilde{S}(-k)\left(\frac{k^{2}}{8 \mu^{2}}\right)^{-s-(1 / 2)}\left(1+\frac{8 M^{2}}{k^{2}}\right)^{-(1 / 2)-s}\left[\frac { \Gamma ( - s ) } { \Gamma ( \frac { 1 } { 4 } - \frac { s } { 2 } ) \Gamma ( \frac { 3 } { 4 } - \frac { s } { 2 } ) } F \left(\frac{s}{2}+\frac{3}{4}, \frac{s}{2}+\frac{1}{4} ; s ; 1\right.\right. \\
& \left.\left.-\frac{k^{4}}{\left(k^{2}+8 M^{2}\right)^{2}}\right)+\left(\frac{-8 M^{2}}{k^{2}}\right)^{-s} \frac{\Gamma(s)}{\Gamma\left(\frac{1}{4}+\frac{s}{2}\right) \Gamma\left(\frac{3}{4}+\frac{s}{2}\right)} F\left(-\frac{s}{2}+\frac{3}{4},-\frac{s}{2}+\frac{1}{4} ; 1-s ; 1-\frac{k^{4}}{\left(k^{2}+8 M^{2}\right)^{2}}\right)\right]
\end{aligned}
$$

Now, for $-1 / 2<\operatorname{Re}(s)<0$, the second term inside the square brackets vanishes when $M=0$, and one gets

$$
\begin{aligned}
\zeta_{2}= & \frac{2^{2 s-2} \mu^{-1} \Gamma\left(s+\frac{1}{2}\right) \Gamma(-s)}{\pi \Gamma(s) \Gamma\left(\frac{1}{2}-s\right)} \\
& \times \int d k \widetilde{S}(k) \tilde{S}(-k)\left(\frac{k^{2}}{\mu^{2}}\right)^{-s-(1 / 2)} .
\end{aligned}
$$

The effective action is then seen to be given by

$$
\begin{aligned}
W^{\mathrm{eff}}= & \frac{1}{2 \pi} \int d k \tilde{S}(k) \tilde{S}(-k)\left(k^{2}\right)^{-1 / 2} \\
& \times\left[2 \gamma+2 \log 2+2 \psi\left(\frac{1}{2}\right)-\log \left(\frac{k^{2}}{\mu^{2}}\right)\right] .
\end{aligned}
$$

Since $\zeta_{2}(s=0) \neq 0$, a dependence on $\log (\mu)$ remains. Comparing with Eq. (23) we see that there is no term of this form in the small $t$ asymptotics of the heat kernel. We con- 
clude that the $\log (\mu)$ term appears due the infrared behavior of the heat kernel (large $t$ ). $\mu$ thus plays the role of an infrared regulator, rather than a renormalization parameter. The corresponding ambiguity cannot be fixed by a normalization condition because this would require the presence of a nonlocal $\left(\partial_{z}^{-1}\right)$ counterterm. Usually, such problems are solved by a resummation of the perturbation theory series. The IR singularity $\mu \rightarrow 0$ in Eq. (45) looks similar to the one which appears in two dimensions on manifolds without boundary. It has been demonstrated in [22] that for this latter case all infrared problems disappear if one uses the dilaton representation [23] for the potential and then collect powers of the dilaton instead of powers of the potential itself.

\section{2. $m=5$}

Here, the limit $M=0$ can be taken directly in Eq. (42), and one easily gets

$$
\begin{aligned}
\zeta_{5} & =\frac{\mu^{2}}{2(4 \pi)^{2}(s-1)} \int d^{4} k \widetilde{S}(k) \widetilde{S}(-k)\left(\frac{k^{2}}{8 \mu^{2}}\right)^{-s+1} F\left(\frac{s}{2}, \frac{s-1}{2} ; 1 ; 1\right) \\
& =\frac{\mu^{2}}{2^{7}(\pi)^{5 / 2}(s-1)} \frac{\Gamma\left(\frac{3}{2}-s\right)}{\Gamma(2-s)} \int d^{4} k \widetilde{S}(k) \widetilde{S}(-k)\left(\frac{k^{2}}{\mu^{2}}\right)^{-s+1} .
\end{aligned}
$$
by

The effective action can be easily obtained, and it is given

$$
\begin{aligned}
W^{\mathrm{eff}}= & -\frac{1}{2^{8}(\pi)^{5 / 2}} \int d^{4} k \widetilde{S}(k) \widetilde{S}(-k) k^{2} \\
& \times\left[2-\gamma-\psi\left(\frac{3}{2}\right)-\log \left(\frac{k^{2}}{\mu^{2}}\right)\right] .
\end{aligned}
$$

This effective action also depends on $\log (\mu)$. But his time, $\log (\mu)$ appears in front of a local action $S \Delta S$. Such a term is present in the small $t$ asymptotics of the heat kernel. Therefore, the $\mu$-dependence appears due to ultraviolet divergences. In principle, the dependence on $\mu$ can be renormalized away provided there is a suitable term in the classical action.

As we have noted after Eq. (4), our results for $m=5$ can be used also in a quantum brane world scenario. Suppose that the scalar field potential on the brane has the usual Higgs form: $\widetilde{V}(\phi) \sim \phi^{4}$. $S$ has to be identified with the second derivative of this potential, $S \sim \overline{\phi^{2}}$. Hence, $S \Delta S \sim \overline{\phi^{2}} \Delta \overline{\phi^{2}}$. Renormalizing this term would require a rather unusual interaction term in the classical action. This is just another example of exotic counterterms [7] which appear in the brane-world scenario. The finite part of the quantum correction goes as $k^{2} \log \left(k^{2}\right)$. Already on dimensional grounds, it is clear that typical scalar theories in four dimensions do not present such strong growth of the effective action at large momenta. Therefore, we come to the (not unexpected) conclusion that the short distance quantum physics depends strongly on the presence of extra dimensions. For more realistic brane models in curved space the heat kernel expansion [7] predicts other $S^{2}$-terms proportional to geometric characteristics of the brane (but independent of $k$ ). These terms will dominate Eq. (47) at small momenta.
Note that in any even dimension greater than $2, \zeta(0)=0$. Thus, no logarithmic operator or dependence on $\mu$ appears in the effective action.

\section{CONCLUSIONS}

In this paper we used the multiple reflection expansion to calculate (parts of) the heat kernel and of the effective action depending on the function $S$ appearing in Robin boundary conditions (3). In particular, we have calculated the Coleman-Weinberg potential on the boundary (assuming constant $S$ ) and the quadratic part in $S$ containing an arbitrary number of tangential derivatives. Applications to tachyon condensation in open string theory and to the braneworld scenario were briefly outlined. More detailed study of these applications will be given elsewhere.

The basic relations (6) and (7) of the multiple reflection expansion method can be extended to other boundary value problems, which correspond to taking more complicated operators instead of just the scalar function $S$. Acting in this way we may cover boundary conditions appearing in the context of Casimir energy calculations [24], solid state physics and strings (see $[25,26]$ for some examples).

\section{ACKNOWLEDGMENTS}

The authors acknowledge support from Fundacin Antorchas and DAAD (grant 13887/1-87). The work of D.V.V. has been supported by the DFG project Bo 1112/11-1. H.F. and E.M.S. also acknowledge CONICET (Argentina) (grant 0459/98).

\section{APPENDIX A: CURVED BOUNDARY}

In this appendix we demonstrate that our results can be extended to the case of a curved boundary. To this end, we use the conformal variation technique, which is quite differ- 
ent from the expansions used in the main text. For simplicity, we neglect derivatives of $S$.

The heat kernel coefficients for Robin boundary conditions are locally computable. This means that they can be expressed through volume and surface integrals of some local invariants. In particular, on dimensional grounds, we can write

$$
\begin{aligned}
a_{n}(f, D) \simeq & (4 \pi)^{-(m-1) / 2} \\
& \times \int_{\partial \mathcal{M}} d z\left(c_{0} S^{n-1}+c_{1} f_{; m} S^{n-2} c_{2} L_{a a} S^{n-2}+\ldots\right),
\end{aligned}
$$

where $L_{a a}$ denotes the trace of the extrinsic curvature of the boundary. We dropped many other invariants which are not relevant for the present calculation. It is important that the constants $c_{k}$ do not depend on $m$.

Let us consider a local conformal transformation of the operator $D: D \rightarrow e^{-2 \epsilon f} D$. All local invariants and the heat kernel coefficients change under this transformation. One can show [15] that

$$
\left.\frac{d}{d \boldsymbol{\epsilon}}\right|_{\epsilon=0} a_{n}\left(1, e^{-2 \epsilon f} D\right)=(m-n) a_{n}(f, D) .
$$

Transformation rules for individual local invariants can be found in [15] (see also [27]). Let us consider the terms which produce $f_{; m} S^{n-2} \quad(n \geqslant 3)$ after conformal variation. Obviously, there are only two such terms:

$$
\begin{gathered}
\left.\frac{d}{d \epsilon}\right|_{\epsilon=0} S^{n-1} \rightarrow \frac{(m-2)(n-1)}{2} f_{; m} S^{n-2} \\
\left.\frac{d}{d \epsilon}\right|_{\epsilon=0} L_{a a} S^{n-2} \rightarrow-(m-1) f_{; m} S^{n-2} .
\end{gathered}
$$

One has to remember that the volume element on the boundary is also changed: $d z \rightarrow e^{(m-1) \epsilon f} d z$.

Collecting the terms containing $f_{; m} S^{n-2}$ on both sides of Eq. (A2), we obtain $(n>2)$ :

$$
\frac{n-1}{2}(m-2) c_{0}-(m-1) c_{2}=(m-n) c_{1} .
$$

Equation (A4) can be solved giving:

$$
c_{1}=c_{0} / 2 .
$$

We can immediately calculate all terms in the heat kernel expansion which are linear in $L_{a b}$ and contain arbitrary power of $S$ :

$$
\begin{aligned}
K(f, t) \simeq & \frac{1}{(4 \pi)^{(m-1) / 2}} \int_{\partial \mathcal{M}} d z f(z) \\
& \times \sum_{l} S^{l}(z) L_{a a}(z) t^{1 / 2(l-m+2)} \frac{l}{4 \Gamma\left(\frac{l+3}{2}\right)} .
\end{aligned}
$$

This is consistent with $[15,28]$ for $l=1,2,3$. As an additional consistency check we see that all dependence on $m$ resides in a power of $4 \pi t$ only.

The right hand side of Eq. (A6) can again be represented through the error function [c.f. Eq. (17)]. All the calculations of Sec. III A can be repeated step by step, thus giving the effective potential on a slightly curved boundary.

\section{APPENDIX B: DISCUSSION OF NEGATIVE EIGENVALUES}

We will, in the first place, prove that the exponentially growing part (29) of the trace of the heat kernel for $S^{2}$ $>M^{2}$ is precisely the contribution due to the eigenvalues of $-\partial^{2}+M^{2}$ eventually becoming negative. Such eigenvalues are of the form

$$
\lambda_{-}=M^{2}-S^{2}+k^{2},
$$

where $k$ is the boundary momentum. Then, their contribution to the trace of the heat kernel is given by

$$
K_{d i v}(t)=\frac{V}{(2 \pi)^{m-1}} \int_{-\infty}^{\infty} d^{m-1} k e^{-\left(M^{2}-S^{2}+k^{2}\right) t},
$$

where the prefactor is the density of states on the boundary.

After making explicit the integration measure, one has

$$
\begin{aligned}
K_{d i v}(t)= & \frac{2 V \pi^{(m-1) / 2}}{(2 \pi)^{m-1} \Gamma\left(\frac{m-1}{2}\right)} \\
& \times \int_{0}^{\infty} d k k^{m-2} e^{-\left(M^{2}-S^{2}+k^{2}\right) t} .
\end{aligned}
$$

By changing the integration variable to $k^{\prime}=k^{2} t$, the final result arises, which is

$$
K_{d i v}(t)=\frac{V e^{\left(S^{2}-M^{2}\right) t}}{(4 \pi t)^{(m-1) / 2}} .
$$

Now, we will determine the contribution of these modes to the zeta function in a direct way. It is given by

$$
\begin{aligned}
\zeta_{2}^{D}(s)= & \frac{2 V \mu^{2 s} \pi^{(m-1) / 2}}{(2 \pi)^{m-1} \Gamma\left(\frac{m-1}{2}\right)} \\
& \times \int_{0}^{\infty} d k k^{m-2}\left(M^{2}-S^{2}+k^{2}\right)^{-s},
\end{aligned}
$$

or, changing variables, 


$$
\zeta_{2}^{D}(s)=\frac{V \mu^{2 s}}{(4 \pi)^{(m-1) / 2} \Gamma\left(\frac{m-1}{2}\right)}\left[(-1)^{-s} \int_{0}^{S^{2}-M^{2}} d k k^{(m-3) / 2}\left(S^{2}-M^{2}-k\right)^{-s}+\int_{S^{2}-M^{2}}^{\infty} d k k^{(m-3) / 2}\left(M^{2}-S^{2}+k\right)^{-s}\right] .
$$

After performing the integrals, one finally gets

$$
\zeta_{2}^{D}(s)=\frac{V \mu^{2 s}\left(S^{2}-M^{2}\right)^{-s+(m-1) / 2}}{(4 \pi)^{(m-1) / 2} \Gamma\left(\frac{m-1}{2}\right)}\left[(-1)^{-s} \frac{\Gamma\left(\frac{m-1}{2}\right) \Gamma(1-s)}{\Gamma\left(\frac{m+1-2 s}{2}\right)}+\frac{\Gamma\left(s+\frac{1-m}{2}\right) \Gamma(1-s)}{\Gamma\left(\frac{3-m}{2}\right)}\right] .
$$

\section{APPENDIX C: COLEMAN-WEINBERG LAGRANGIANS}

\section{FOR $M \neq 0$}

$$
\text { 1. } S^{2}<M^{2}
$$

For $m=1$

$$
\mathcal{L}^{\text {eff }}=\frac{1}{2} \log \left(\frac{M-S}{\mu}\right)
$$

The dependence on $\mu$ can be renormalized away by requiring that the effective Lagrangian vanishes when $M \rightarrow \infty$. In this case, this is equivalent to subtracting a field-independent term, which is merely a redefinition of the cosmological constant. After doing so, one gets

$$
\mathcal{L}^{\text {eff }}=\frac{1}{2} \log \left(1-\frac{S}{M}\right)
$$

For $m=2$

$$
\begin{aligned}
\mathcal{L}^{\text {eff }}= & \frac{S}{4 \pi}\left[-2+\log \frac{M^{2}}{\mu^{2}}+2 \frac{\sqrt{M^{2}-S^{2}}}{S}\right. \\
& \left.\times \arctan \left(\frac{S}{\sqrt{M^{2}-S^{2}}}\right)\right]+\frac{1}{4} \sqrt{M^{2}-S^{2}} .
\end{aligned}
$$

The dependence on $\mu$ can again be eliminated by asking the effective Lagrangian to vanish in the infinite mass limit. But, in this case, this amounts to renormalizing a term linear in $S$.

For $m=3$

$$
\begin{aligned}
\mathcal{L}^{\mathrm{eff}}= & \frac{1}{16 \pi}\left[M^{2}-S^{2}+2 M S\right. \\
& \left.-2\left(M^{2}-S^{2}\right) \log \left(\frac{M-S}{\mu}\right)\right] .
\end{aligned}
$$

For $m=4$

$$
\begin{aligned}
\mathcal{L}^{\mathrm{eff}}= & \frac{-1}{144 \pi^{2}}\left[16 S^{3}-21 M^{2} S+6 \pi\left(M^{2}-S^{2}\right)^{3 / 2}\right. \\
& +6\left(3 M^{2} S-2 S^{3}\right) \log \left(\frac{M}{\mu}\right) \\
& \left.+12\left(M^{2}-S^{2}\right)^{3 / 2} \arctan \left(\frac{S}{\sqrt{M^{2}-S^{2}}}\right)\right] .
\end{aligned}
$$

For $m=5$

$$
\begin{aligned}
& \mathcal{L}^{\mathrm{eff}}= \frac{1}{768 \pi^{2}}\left[-9 M^{4}-20 M^{3} S+18 M^{2} S^{2}\right. \\
&\left.+12 M S^{3}-9 S^{4}+12\left(M^{2}-S^{2}\right)^{2} \log (M-S)\right] . \\
& \text { 2. } S^{2}>M^{2}, S>0
\end{aligned}
$$

For $m=1$

$$
\mathcal{L}^{\mathrm{eff}}=-\frac{1}{2} \log \left(\frac{S^{2}-M^{2}}{\mu^{2}}\right)+\arg \tanh \left(\frac{M}{S}\right) \pm i \pi .
$$

$$
\text { 3. } S^{2}>M^{2}, S<0
$$

For $m=1$

$$
\mathcal{L}^{\mathrm{eff}}=-\frac{1}{2} \log \left(\frac{S^{2}-M^{2}}{\mu^{2}}\right)-\arg \tanh \left(\frac{M}{|S|}\right)
$$

[1] A. Sen, J. High Energy Phys. 08, 012 (1998); A. Sen and B. Zwiebach, ibid. 03, 002 (2000).

[2] K. Bardakci, Nucl. Phys. B68, 331 (1974); V.A. Kostelecky and S. Samuel, ibid. B336, 263 (1990).
[3] K. Ohmori, "A review on tachyon condensation in open string field theories," hep-th/0102085.

[4] A.A. Tseytlin, J. Math. Phys. 42, 2854 (2001).

[5] L. Randall and R. Sundrum, Phys. Rev. Lett. 83, 3370 (1999); 
83, 4690 (1999); V.A. Rubakov and M.E. Shaposhnikov, Phys. Lett. 125B, 136 (1983).

[6] M. Bordag and D.V. Vassilevich, J. Phys. A 32, 8247 (1999).

[7] P.B. Gilkey, K. Kirsten, and D.V. Vassilevich, Nucl. Phys. B601, 125 (2001).

[8] P. Minces and V.O. Rivelles, Nucl. Phys. B572, 651 (2000); J. High Energy Phys. 12, 010 (2001).

[9] P. B. Gilkey, Invariance Theory, the Heat Equation, and the Atiyah-Singer Index Theorem (CRC, Boca Raton, FL, 1995); G. Esposito, A. Yu. Kamenshchik, and G. Pollifrone, Euclidean Quantum Gravity on Manifolds with Boundary (Kluwer, Dordrecht, 1997); I. Avramidi, Heat Kernel and Quantum Gravity (Springer, Berlin, 2000); K. Kirsten, Spectral Functions in Mathematics and Physics (CRC, Boca Raton, FL, 2001); I. L. Buchbinder, S. D. Odintsov, and I. L. Shapiro, Effective Action in Quantum Gravity (IOP, Bristol, 1992).

[10] M. Bordag, D.V. Vassilevich, H. Falomir, and E.M. Santangelo, Phys. Rev. D 64, 045017 (2001).

[11] R. Balian and C. Bloch, Ann. Phys. (N.Y.) 60, 401 (1970); 85, 514 (1974); R. Balian and B. Duplantier, ibid. 104, 300 (1977); T.H. Hansson and R.L. Jaffe, ibid. 151, 204 (1983).

[12] W. Kummer and D.V. Vassilevich, J. High Energy Phys. 07, 012 (2000).

[13] I.G. Moss, Phys. Lett. B 491, 203 (2000).

[14] A. Romeo and A.A. Saharian, "Casimir effect for scalar fields under Robin boundary conditions on plates," hep-th/0007242.

[15] T.P. Branson and P.B. Gilkey, Commun. Partial Diff. Eq. 15, 245 (1990).

[16] T.P. Branson, P.B. Gilkey, and D.V. Vassilevich, Boll. Unione Mat. Ital. 11B, 39 (1997).

[17] T.P. Branson, P.B. Gilkey, K. Kirsten, and D.V. Vassilevich, Nucl. Phys. B563, 603 (1999).

[18] S.M. Blinder, Phys. Rev. A 37, 973 (1988).

[19] H. S. Carslaw and J. C. Jaeger, Conduction of Heat in Solids, 2nd ed. (Oxford University Press, Oxford, 1959).

[20] J.S. Dowker and R. Critchley, Phys. Rev. D 13, 3224 (1976).

[21] S.W. Hawking, Commun. Math. Phys. 55, 133 (1977).

[22] Y.V. Gusev and A.I. Zelnikov, Phys. Rev. D 61, 084010 (2000).

[23] V. Mukhanov, A. Wipf, and A. Zelnikov, Phys. Lett. B 332, 283 (1994); W. Kummer and D.V. Vassilevich, Phys. Rev. D 60, 084021 (1999).

[24] M. Bordag, U. Mohideen, and V.M. Mostepanenko, Phys. Rep. 353, 1 (2001).

[25] M. Bordag and D.V. Vassilevich, Phys. Lett. A 268, 75 (2000).

[26] D.V. Vassilevich, Nucl. Phys. B (Proc. Suppl.) 104, 208 (2002).

[27] D.V. Vassilevich, J. Math. Phys. 36, 3174 (1995).

[28] K. Kirsten, Class. Quantum Grav. 15, L5 (1998). 